\title{
Comparative Study of a Single-Incision Laparoscopic and a Conventional Laparoscopic Appendectomy for the Treatment of Acute Appendicitis
}

\author{
Jungwoo Kang, Byung Noe Bae ${ }^{1}$, Geumhee Gwak ${ }^{1}$, Inseok Park ${ }^{1}$, Hyunjin Cho ${ }^{1}$, Keunho Yang ${ }^{1}$, \\ Ki Whan Kim ${ }^{1}$, Sehwan Han ${ }^{1}$, Hong-Joo Kim ${ }^{1}$, Young-Duck Kim ${ }^{1}$ \\ Department of Surgery, Inje University Seoul Paik Hospital, ${ }^{1}$ Inje University Sanggye Paik Hospital, Seoul, Korea
}

Purpose: For the treatment of acute appendicitis, a conventional laparoscopic appendectomy (LA) has been widely performed. Recently, the use of single incision laparoscopic surgery (SILS) is increasing because it is believed to have advantages over conventional laparoscopic surgery. In this study, we compared SILS and a conventional LA.

Methods: We analyzed the 217 patients who received laparoscopy-assisted appendectomies between August 2010 and April 2012 at Inje University Sanggye Paik Hospital. One hundred-twelve patients underwent SILS, and 105 patients underwent LA. For the two groups, we compared the operation times, postoperative laboratory results, postoperative pain, hospital stay, and postoperative complications.

Results: The patients' demographics, including body mass index, were not significantly different between the two groups. There were 6 perforated appendicitis cases in the SILS group and 5 cases in the LA group. The mean operative time in the SILS group was $65.88 \pm 22.74$ minutes whereas that in the LA group was $61.70 \pm 22.27$ minutes $(P=0.276)$. There were no significant differences in the mean hospital stays, use of nonsteroidal antiinflammatory drugs, and wound infections between the two groups.

Conclusion: Postoperative pain, complications and hospital stay showed no statistically significant differences between the SILS and the LA groups. However, our SILS method uses a single trocar and two latex tubes, so cost savings and reduced interference during surgery are expected.

Keywords: Single port; Laparoscope; Appendectomy

\section{INTRODUCTION}

The laparoscopic appendectomy (LA) was first described by Semm [1], a German gynecologist, in 1983. Pier et al. [2] presented their study comparing a LA and an open appendectomy in Germany in 1991, and they reported the advantages of a LA by comparing it with a conventional appendectomy. Unlike an open appendec-

Received: July 28, 2012 - Accepted: October 29, 2012

Correspondence to: Byung Noe Bae, M.D.

Department of Surgery, Inje University Sanggye Paik Hospital,

1342 Dongil-ro, Nowon-gu, Seoul 139-707, Korea

Tel: +82-2-950-1023, Fax: +82-2-3391-4393

E-mail: bnbae@paik.ac.kr

(C) 2012 The Korean Society of Coloproctology

This is an open-access article distributed under the terms of the Creative Commons Attribution NonCommercial License (http://creativecommons.org/licenses/by-nc/3.0) which permits unrestricted non-

commercial use, distribution, and reproduction in any medium, provided the original work is properly cited. tomy, a LA not only minimizes tissue damage but also decreases the risk of potential postoperative adhesion by reducing intestinal stimulation. Moreover, it shortens the hospital stay by enabling early ambulation, early oral nutrition, etc. [3-5]. In addition to its aesthetic effect, lessened pain, and shorter hospital stay, a LA enables the diseased appendix and other abdominal diseases to be easily and swiftly detected and diminishes the risks of postoperative intestinal adhesion and surgical wound infection $[6,7]$. In cases of perforated appendicitis, in particular, associated damage to other abdominal organs can be detected. Finally, the LA is more favorable in terms of fasting period, hospital admissions, and the presence of complications, with inflammatory discharge in the abdominal cavity being washed away [8].

Along with recent advances in surgical instruments and techniques, single-incision laparoscopic surgery (SILS) and natural orifice transluminal endoluminal surgery are experimentally being performed for cosmetic improvements. The authors of this 
Volume 28, Number 6, 2012

study were able to reduce the required three or more surgical incisions to a single incision in a LA. We chose to perform SILS because its operative techniques are similar to those of a conventional LA and are easily adaptable [9]. In this regard, the study aimed to examine the usefulness of SILS by comparing SILS performed in our hospital with a conventional LA. Furthermore, the study investigated the effectiveness of the surgical glove developed for single-incision insertion.

\section{METHODS}

\section{Study population}

The study comprised patients who underwent SILS $(\mathrm{n}=112)$ or a conventional LA $(n=105)$ conducted by the same two surgeons. The study included patients who were admitted to the Surgery Department of Inje University Sanggye Paik Hospital due to acute appendicitis from August 2010 to April 2012. A single surgeon performed surgery on 113 of the patients, and the other surgeon performed surgery on 104 of the patients. Subjects were selected from all appendicitis patients treated at our hospital during the work hours of the two surgeons. The operative techniques were selected randomly regardless of gender and age of patients and were performed in almost identical ratios. Medical records were used to conduct a retrospective comparative analysis of sex, age, body mass index (BMI), operation time, admission days, the number of painkillers injected, the presence of complications, etc. Subjects were diagnosed based on history taking, physical examination, abdominal ultrasonography, and abdominal computed tomography scan.

\section{Surgical methods}

Surgery was performed to all patients after the insertion of a Foley catheter under general anesthesia. In the case of a LA, a veress nee-

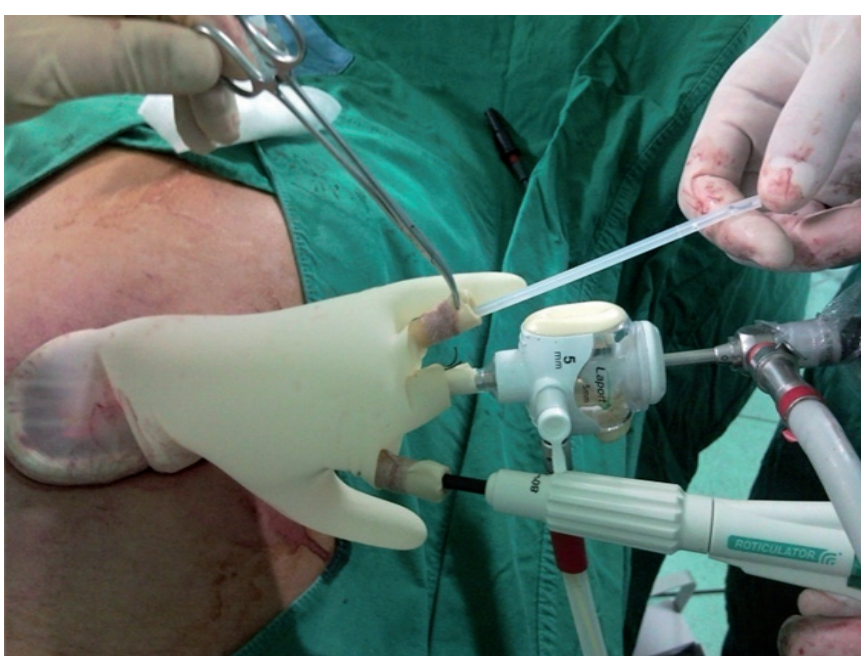

Fig. 1. Multichannel single port was created using a surgical glove, one trocar, two 7 Fr. Latex tubes in the operation field. dle was inserted into the abdominal cavity by making a perpendicular incision in the navel area. A $10-\mathrm{mm}$ trocar was inserted into the incision site with a raised intraabdominal pressure of 10 to $12 \mathrm{mmHg}$. A $30^{\circ}, 5-\mathrm{mm}$ laparoscope was inserted to visualize the abdominal cavity. A 5-mm trocar was carefully inserted, relying on the laparoscopic light source and avoiding contact with the abdominal wall vessels, to the suprasphincteric portion of the pubis on both sides of the lower abdomen. Patients were tilted in left lateral positions to $15^{\circ}$ to $20^{\circ}$ to secure easy access to the appendix. Subsequently, the LA was performed using procedures identical to those of conventional laparoscopic surgery.

In the case of SILS, an approximately $1.5-\mathrm{cm}$ perpendicular incision was made at the naval. A wound retractor (ALEXIS O small wound retractor, Applied Medical, Rancho Santa Margarita, CA, USA) was inserted, and the incision site was covered with surgical gloves No. 6. The index and ring fingers of the surgical gloves No. 6 were cut about $1 \mathrm{~cm}$, and a 7 Fr. Latex tube was fixed with sterilized paper tape to replace the trocars. After cutting off about $1 \mathrm{~cm}$ of the middle finger of the surgical glove, a 5-mm trocar was inserted (Fig. 1). A laparoscopic camera was inserted through the trocar of the middle finger, and surgery was carried out by inserting other instruments through the index and the ring fingers. Using a $30^{\circ}, 5-\mathrm{mm}$ camera, we visualized the location of the appendix by using two straight instruments or a straight instrument combined with a flexible instrument. Next, the mesoappendix was dissected after electrocautery, and, if needed, vessels were ligated using ultrasonic shear (Sonosurg, Olympus Co., Tokyo, Japan). Subsequently, the exposed basal appendix was ligated using two endoloops (3.5 metric, Ethicon Inc., Somerville, NJ, USA) and cut with laparoscopic scissors. The resected appendix was taken out through the navel; then, the site was tied with a knot by using the thumbs of surgical gloves No. 6 (Fig. 2). Surgical sites were washed with normal saline, if needed, after bleeding, complications such as in-

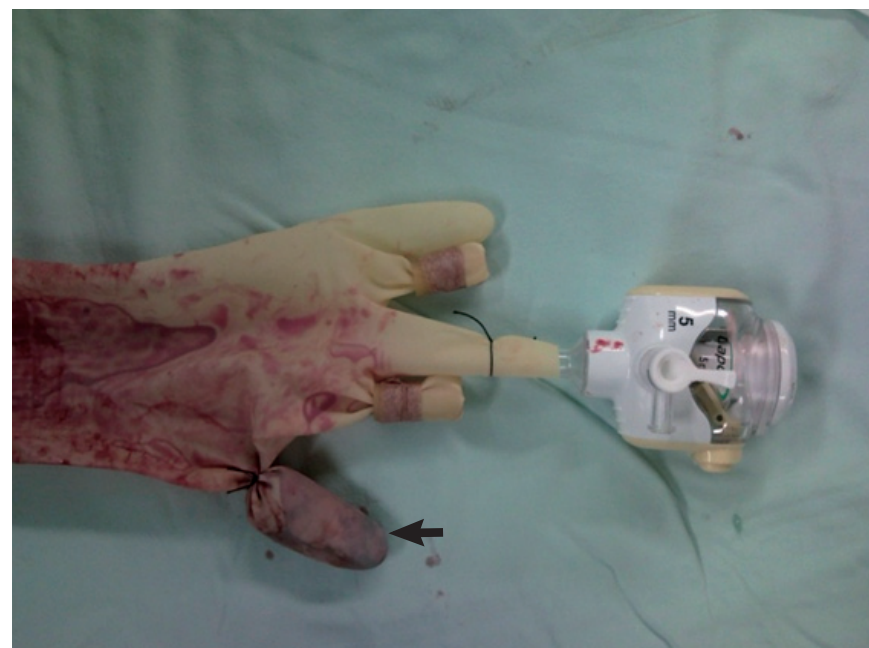

Fig. 2. Multichannel port when operation was done. Appendix was inserted in the thumb site (arrow). 


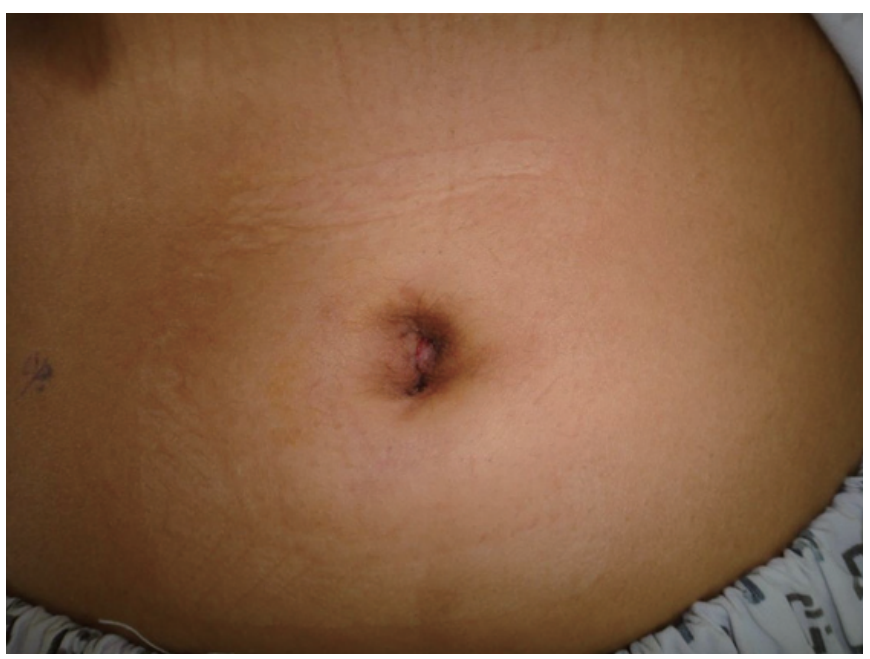

Fig. 3. Operation wound on postoperative day \#7.

testinal perforation in the abdominal cavity, and abnormal conditions in peripheral organs had been confirmed. The two operative techniques showed no significant differences other than the number of surgical incisions.

\section{RESULTS}

\section{Comparison of clinical characteristics of subjects}

Among the total of 217 patients, LA and SILS were performed on 105 (48.4\%) and 112 patients (51.6\%), respectively. The mean age was slightly higher in the SILS group, and the number of male patients was higher in both groups. The average BMI was slightly higher in the LA group, and the mean preoperative white blood cells were slightly higher in the SILS group. Howerever, Our study found no statistically significant differences between LA group and SILS group (Table 1).

\section{Operations and complications}

The length of hospital stay for the LA group was slightly longer than it was for the SILS group, but the difference was not statistically significant. The number of injected painkillers was also lower in the SILS group, but no statistical difference was found. There was also neither significant difference nor statistical significance in postoperatively measured visual analogue scale (VAS). Although a higher number of patients with perforated appendicitis received SILS, no statistical significance was shown compared with a LA. Even though SILS decreased the overall incidence of surgical site infection, no statically significant results were found. When the total operation time was defined as the time from the moment of skin incision to the point of successfully completing wound closure, no statistically significance differences were observed in spite of the operation time for SILS being longer $(\mathrm{P}=0.276)$ (Table 2). The postoperative surgical wound was hidden by the wrinkles of navel and was checked after the first postoperative week on an out-
Table 1. Characteristics of patients

\begin{tabular}{lccc}
\hline Characteristic & LA $(\mathrm{n}=105)$ & SILS $(\mathrm{n}=112)$ & P-value \\
\hline Age $(\mathrm{yr})$ & $30.5 \pm 16.5$ & $32.8 \pm 18.0$ & 0.756 \\
Gender (male:female) & $60: 45$ & $58: 54$ & 0.496 \\
BMI $\left(\mathrm{kg} / \mathrm{m}^{2}\right)$ & $22.2 \pm 4.0$ & $22.1 \pm 3.8$ & 0.838 \\
Preoperative-WBCs $(/ \mu \mathrm{L})$ & $12,708 \pm 4,012$ & $12,915 \pm 4,627$ & 0.550
\end{tabular}

Values are presented as mean \pm standard deviation.

LA, laparoscopic appendectomy; SILS, single incision laparoscopic surgery; BMI, body mass index; WBC, white blood cell.

Table 2. Operative and postoperative clinical data

\begin{tabular}{lccc}
\hline & LA $(\mathrm{n}=105)$ & $\operatorname{SILS}(\mathrm{n}=112)$ & P-value \\
\hline Hospital stay (day) & $3.05 \pm 2.39$ & $2.89 \pm 2.41$ & 1.000 \\
Use of NSAIDs & $5.51 \pm 2.98$ & $5.00 \pm 1.84$ & 1.000 \\
VAS (postoperative) & $5.14 \pm 1.48$ & $5.02 \pm 1.35$ & 0.135 \\
Wound infection & 9 & 6 & 0.427 \\
Operation time (min) & $61.70 \pm 22.27$ & $65.88 \pm 22.74$ & 0.276 \\
Perforation & 5 & 6 & 1.000 \\
\hline
\end{tabular}

Values are presented as mean \pm standard deviation.

LA, laparoscopic appendectomy; SILS, single incision laparoscopic surgery; NSAIDs, nonsteroidal antiinflammatory drugs; VAS, visual analogue scale.

patient basis (Fig. 3).

\section{DISCUSSION}

SILS requires a laparoscopic camera and two straight instruments through a single incision. For this reason, interferences and collisions between surgical instruments are worse than they are when a LA is performed using three incisions. Moreover, securing a surgical view is problematic due to parallel placements of the camera and the instruments [10]. In the early stage of SILS, such challenges extended the operation time and caused difficulties during surgical procedures. However, those problems in surgical techniques were overcome by accumulated experience, allowing a shorter operating time to be achieved, as has already been proved by the learning curve reported in a study conducted during the initial stage of SILS at our hospital [11].

Teoh et al. [12] reported that no statistically significant difference was detected in the operation time and admission days between the SILS and the LA groups. This study also could not find any statistically significant differences in admission days between the two groups. Taking into consideration the fact that three surgical instruments need to be inserted through a single incision at the same time in the case of SILS, a larger incision in the navel, than in the LA technique, is required. Hence, more intense pain or surgical wound infection is anticipated in the SILS technique. Based on such a theoretical basis, Kim et al. [10] reported that more intense pain was generated with the SILS technique than with the 
LA technique. On the other hand, Mayer et al. [13] reported that the pain was less severe postoperatively in the SILS group compare with the LA group. No differences were shown between the two groups during a study on pain management regarding wound infection and the number of VAS and painkiller injections.

Amos et al. [14] concluded that no specific benefits were found in the SILS and the LA techniques because almost identical results were obtained for the operation time, intraoperative bleeding, and admission days of the two groups. Park et al. [15] also suggested that no statistical differences were present between the LA and the SILS groups when the operation time and the admission days were compared. This study also detected no statistical differences in operation time and admission days between the two groups.

Some reports have stated that the use of flexible laparoscopic instruments instead of straight instruments may reduce the collisions between instruments [16]. Although our hospital also initially used flexible laparoscopic instruments, with accumulated experience, only straight laparoscopic instruments are currently used. A camera was placed in the center of the incision hole, and angles were adjusted by holding the instruments at both lateral sides. This enabled the resection of the mesoappendix and the appendix without laparoscopic instruments. According to other studies on SILS, three trocars in three fingers of a surgical glove were placed through a single incision. However, our study came up with measures that could diminish the interferences and collisions generated due to trocars by inserting a single trocar and two tubes through the surgical glove. In the case of using three trocars, the surgical site could be limited due to collisions of the three trocars. When the three trocars were replaced with two thin tubes with a single trocar, the collision of trocars was minimized, and surgical procedures were successfully carried out without much problem.

This comparative study analyzed a large number of patients in a relatively short period of time. Two specialized surgeons performed SILS or the LA on all appendicitis patients treated in our hospital during the work hours of the surgeons. Unlike a prospective randomized study, this study has some limitations in that it was not conducted based on a statistical hypothesis with calculation and management in advance. In addition, the potential bias of different surgeons could be another limitation.

In conclusion, no significant differences were found when operation time and postoperative pain were compared between the SILS and the LA groups. In addition, because of insufficient results, we failed to find statistically significant differences between the two groups for complications other than wound infections. Except for the number of painkillers injected, the study was conducted without precise objective indicators for the degree of pain. Thus, supplementary studies need to be performed in the future to acquire more objective results.

SILS and a LA showed not much difference in relation to surgical outcomes and performance, but SILS was more effective in decreasing the risk of postoperative wound infection. However, other operative difficulties of single-incision surgery need to be overcome by the surgeon. In addition, the glove-port procedure used in the study is thought to be more effective than conventional SILS procedures while the use of a single trocar minimizes instrumental interference and is beneficial in terms of cost.

\section{CONFLICT OF INTEREST}

No potential conflict of interest relevant to this article was reported.

\section{ACKNOWLEDGMENTS}

This research was supported by 2009 Inje University Research grant.

\section{REFERENCES}

1. Semm K. Endoscopic appendectomy. Endoscopy 1983;15:59-64.

2. Pier A, Gotz F, Bacher C. Laparoscopic appendectomy in 625 cases: from innovation to routine. Surg Laparosc Endosc 1991;1:8-13.

3. Valla JS, Limonne B, Valla V, Montupet P, Daoud N, Grinda A, et al. Laparoscopic appendectomy in children: report of 465 cases. Surg Laparosc Endosc 1991;1:166-72.

4. Masoomi H, Nguyen NT, Dolich MO, Wikholm L, Naderi N, Mills $S$, et al. Comparison of laparoscopic versus open appendectomy for acute nonperforated and perforated appendicitis in the obese population. Am J Surg 2011;202:733-8.

5. Varlet F, Tardieu D, Limonne B, Metafiot H, Chavrier Y. Laparoscopic versus open appendectomy in children: comparative study of 403 cases. Eur J Pediatr Surg 1994;4:333-7.

6. Ablassmaier B, Kiessling S, Pier A. "Open" versus laparoscopic appendectomy. Chirurg 1996;67:522-5.

7. Sauerland S, Lefering R, Holthausen U, Neugebauer EA. Laparoscopic vs conventional appendectomy: a meta-analysis of randomised controlled trials. Langenbecks Arch Surg 1998;383: 289-95.

8. Lin HF, Wu JM, Tseng LM, Chen KH, Huang SH, Lai IR. Laparoscopic versus open appendectomy for perforated appendicitis. J Gastrointest Surg 2006;10:906-10.

9. Kim JW, Park JS, Chang IT, Choi YS, Song HJ, Kim BG. The initial experience with a single incision laparoscopic appendectomy. J Korean Soc Coloproctol 2009;25:312-7.

10. Kim HO, Yoo CH, Lee SR, Son BH, Park YL, Shin JH, et al. Pain after laparoscopic appendectomy: a comparison of transumbilical single-port and conventional laparoscopic surgery. J Korean Surg Soc 2012;82:172-8.

11. Kang JW, Bae BN, Guak GH, Yang KH, Kim KH, Han S, et al. A comparetive study with single incision laparoscopic surgery and traditional laparoscopic technique in acute appendicitis [abstract SP-11]. In: 10th meeting of endoscopic and laparoscopic surgeons of ASIA; 2010 Nov 25-27; Hanoi, Vietnam. Chai Wan: ELSA; 2010;226.

12. Teoh AY, Chiu PW, Wong TC, Wong SK, Lai PB, Ng EK. A case- 
controlled comparison of single-site access versus conventional three-port laparoscopic appendectomy. Surg Endosc 2011;25: 1415-9.

13. Mayer S, Werner A, Wachowiak R, Buehligen U, Boehm R, Geyer $\mathrm{C}$, et al. Single-incision multiport laparoscopy does not cause more pain than conventional laparoscopy: a prospective evaluation in children undergoing appendectomy. J Laparoendosc Adv Surg Tech A 2011;21:753-6.

14. Amos SE, Shuo-Dong W, Fan Y, Tian Y, Chen CC. Single-incision versus conventional three-incision laparoscopic appendectomy: a single centre experience. Surg Today 2012;42:542-6.

15. Park J, Kwak H, Kim SG, Lee S. Single-port laparoscopic appendectomy: comparison with conventional laparoscopic appendectomy. J Laparoendosc Adv Surg Tech A 2012;22:142-5.

16. Kang DB, Lee SH, Lee SY, Oh JT, Park DE, Lee C, et al. Application of single incision laparoscopic surgery for appendectomy in children. J Korean Surg Soc 2012;82:110-5. 